\title{
Optical manipulation of collective spin correlations in semiconductors with a squeezed vacuum of polarized photons
}

\author{
E. Ginossar, * Y. Levinson, and S. Levit \\ Department of Condensed Matter Physics, The Weizmann Institute of Science, Rehovot 76100, Israel
}

(Received 25 September 2007; published 7 January 2008)

\begin{abstract}
We calculate the transfer rate of correlations from polarization entangled photons to the collective spin of a many-electron state in a two-band system. It is shown that when a semiconductor absorbs pairs of photons from a two-mode squeezed vacuum, certain fourth-order electron-photon processes correlate the spins of the excited electron pairs of different quasimomenta. Different distributions of the quantum Stokes vector of the light lead to either enhancement or reduction of the collective spin correlations, depending on the symmetry of the distribution. We find that as the squeezing of the light becomes nonclassical, the spin correlations exhibit a crossover from being positive with an $\sim N^{2}$ ( $N$ is the average photon number) scaling to being negative with $\sim N$ scaling, even when $N$ is not small. Negative spin correlations mean a preponderance of spin singlets in the optically generated state. We discuss the possibility to measure the collective spin correlations through the measurement of the Faraday rotation fluctuation spectrum in a steady-state configuration.
\end{abstract}

DOI: 10.1103/PhysRevB.77.035307

PACS number(s): 78.67.De, 42.50.Dv, 42.50.Lc, 42.55.Sa

\section{INTRODUCTION}

Optical excitation of a semiconductor with circularly polarized light generates an average collective spin polarization $\langle\mathbf{S}(t)\rangle$ in the conduction band, also known as optical orientation. ${ }^{1-3}$ This allows us to investigate spin relaxation mechanisms in semiconductors using techniques such as time-resolved Faraday rotation and time-resolved photoluminescence. $^{4-7}$ In this work, we study the optical generation of spin correlations in bulk semiconductors using polarization squeezed light. This can be seen as either an extension of optical orientation to manipulate the fluctuations of the spin $\left\langle\mathbf{S}(t) \cdot \mathbf{S}\left(t^{\prime}\right)\right\rangle$ or as a way to transfer correlations from the light to the electrons. Early theoretical suggestions employing squeezed light involved models with radiation reservoirs interacting with atoms ${ }^{8,9}$ and recently for semiconductors. ${ }^{10}$ However, these are very difficult to implement experimentally. Schemes for transferring correlations from light to matter have recently been explored in atomic and molecular optics (AMO) both theoretically ${ }^{11-13}$ and experimentally. ${ }^{14-16}$ These schemes employ either coherent optical dipoles of atomic $V$ systems or ground states coherence of $\Lambda$ systems, leading to a second-order dependence of the spin fluctuations on the squeezed optical field. In semiconductors, one is faced with strong dephasing of optical dipoles as well as valence band spins due to Coulomb, electron-phonon, and spin-orbit interactions, rendering the above atomic optics schemes impractical. In addition, continuous energy bands in semiconductors have very different level structure and optical selection rules.

In this paper, we suggest to exploit the fact that the dephasing of electronic spins in the conduction band ${ }^{4,17-21}$ is relatively weak. We study spin correlations induced by absorption of photon pairs, a process which does not involve the fast dephasing interband dipoles. We show that due to the increased number of electronic states, there is a large phase space for absorption of photon pairs where each pair is from a different mode. Unlike the AMO case, these processes are fourth order in the coupling to the squeezed optical field. This absorption leads to pairwise spin correlations which are quadratic in the photon occupation. These correlations are generated even when the light is unsqueezed. Absorption of polarization squeezed photon pairs will generate additional pairwise correlations which are also quadratic in the photon correlation. High order effects in the light-matter coupling have been measured in semiconductors, for example, four and six wave-mixing experiments. ${ }^{22,23}$ Here, we find that the spin-spin correlations are enhanced or reduced depending on the correlations of optical modes with different wavelengths and either the same or different polarizations. In particular, it is necessary to use squeezed light in its nonclassical regime in order to generate anticorrelated spins. This is due to a competition between two fourth-order processes inducing positive and negative correlations, the latter becoming dominant only for nonclassical light. In this special regime, the spin correlations depend linearly on the photon correlation, a direct result of the noncommutativity of the photon operators.

\section{MODEL}

Specifically, we are interested in the variance $\left\langle\mathbf{S}^{2}(t)\right\rangle$ of the photoexcited conduction band spin fluctuations, which can be expanded in powers of the optical field up to fourth order $\left\langle\mathbf{S}^{2}(t)\right\rangle=\left\langle\mathbf{S}^{2}(t)\right\rangle^{(2)}+\left\langle\mathbf{S}^{2}(t)\right\rangle^{(4)}$. We show that $\left\langle\mathbf{S}^{2}(t)\right\rangle^{(4)}$ is the lowest order contribution sensitive to photon correlations and calculate its optical generation rate $C_{s}$. Since for semiconductors the spin relaxation rate $\gamma_{s}$ is small relative to other kinetic rates, we expect the steady-state contribution $C_{s} / \gamma_{s}$ to be experimentally measurable.

Polarization properties of photons are described by the quantum Stokes parameters ${ }^{24,25}$ which in the circular polarization basis are written as 


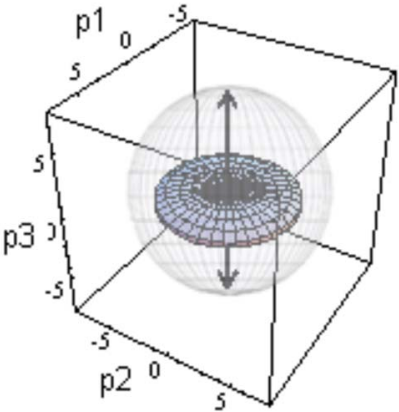

(a)

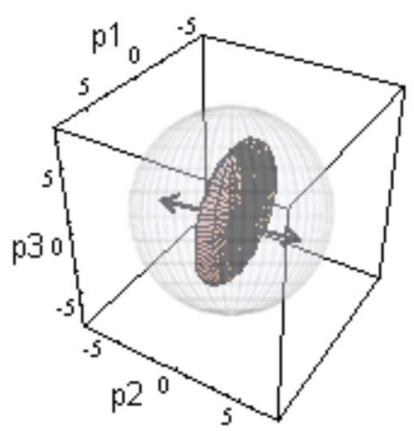

(b)
FIG. 1. (Color online) Covariance ellipsoids for the two generic fluctuation patterns of the Stokes vector $\left(p_{1}, p_{2}, p_{3}\right)$. (a) Oppositepolarization squeezing (for average parameters $N=1, M_{ \pm}^{(1)}=0$, and $\left.M_{ \pm}^{(2)}=1.314\right)$ and (b) same-polarization squeezing $\left(N=1, M_{ \pm}^{(1)}\right.$ $=1.314$, and $\left.M_{ \pm}^{(2)}=0\right)$. The value of $M$ was chosen to be slightly below the maximal squeezing $\sqrt{N(N+1)}$.

$$
\hat{p}_{i}=\sum_{q, q^{\prime}, \lambda, \lambda^{\prime}} b_{q \lambda}^{\dagger}\left(\sigma_{i}\right)_{\lambda \lambda^{\prime}} b_{q^{\prime} \lambda^{\prime}}
$$

where $\sigma_{i=0, \ldots, 3}$ denote the unit and Pauli spin matrix operators and $b_{q \lambda}$ are photon annihilation operators with wave number $q$ and polarization $\lambda$. We consider a collinear pump beam with a range of frequencies $\omega_{0} \pm B / 2$ above the electron-hole gap. We assume that the average Stokes parameters in a given bandwidth $B$ are $\left\langle\hat{p}_{0}\right\rangle=(2 \pi c / L B) \Sigma_{q \lambda} N_{q \lambda}$ and $\left\langle\hat{p}_{1}\right\rangle=\left\langle\hat{p}_{2}\right\rangle=\left\langle\hat{p}_{3}\right\rangle=0$, where $N_{q \lambda}$ is the average photon occupation per mode and $L$ is the quantization length. This describes an unpolarized light with the Stokes vector fluctuating around the origin of the Poincare sphere. The fluctuations are described by the covariance matrix $p_{i j}=1 / 2\left\langle\hat{p}_{i} \hat{p}_{j}+\hat{p}_{j} \hat{p}_{i}\right\rangle$, which for a Gaussian-type field depends on the normal $\left\langle b_{q \lambda}^{\dagger} b_{q^{\prime} \lambda^{\prime}}\right\rangle$ as well as anomalous $\left\langle b_{q \lambda} b_{q^{\prime} \lambda^{\prime}}\right\rangle$ correlations, the latter constituting the main characteristics of squeezed vacuum. ${ }^{26}$ Beams with such properties are generated using the parametric downconversion. ${ }^{27,28}$ In addition to normal correlations $\left\langle b_{q \lambda}^{\dagger} b_{q^{\prime} \lambda^{\prime}}\right\rangle=N_{q} \delta_{\lambda \lambda^{\prime}} \delta_{q q^{\prime}}$, they possess two generic anomalous correlations: same-polarization squeezing $\left\langle b_{q \pm} b_{q^{\prime} \pm}\right\rangle=M_{q \pm}^{(1)} \delta_{q+q^{\prime}, 2 q_{0}} \delta_{\omega_{q+\omega_{q^{\prime}}, 2 \omega_{0}}}$ and opposite-polarization squeezing $\left\langle b_{q \pm} b_{q^{\prime} \mp}\right\rangle=M_{q \pm}^{(2)} \delta_{q+q^{\prime}, 2 q_{0}} \delta_{\omega_{q^{+}}+\omega_{q^{\prime}}, 2 \omega_{0}}$, where $M_{q \pm}^{(1,2)}$ are complex functions and $\omega_{0}=c q_{0}$. It is instructive to draw the covariance ellipsoids for the tensor $p_{i j}$, shown in Fig. 1, for the two cases of the same-polarization and oppositepolarization squeezings based on bandwidth averaged occupation and squeezing functions $N=\bar{N}_{q}$ and $M_{ \pm}^{(1,2)}=\bar{M}_{q \pm}^{(1,2)}$. For squeezed vacuum, $\left\langle\hat{p}_{0} \hat{p}_{1,2,3}\right\rangle=0$, and the fluctuations of $\hat{p}_{1,2,3}$ can be described separately from the variance of $\hat{p}_{0}$. On the axes are plotted $p_{i}$, possible values of $\hat{p}_{i}$. The variance $p_{33}$ given by $2\left[N(N+1)+\left|M^{(1)}\right|^{2}-\left|M^{(2)}\right|^{2}\right]$ for $\left|M_{+}^{(1,2)}\right|=\left|M_{-}^{(1,2)}\right|$ indicating that correlations of the type $M^{(1)}\left(M^{(2)}\right)$ enhance (reduce) the variance $p_{33}$, a fact which is important for spinspin correlations.
The free part of the Hamiltonian of the semiconductor is modeled as a two-band system,

$$
H_{0}=\sum_{q \lambda} \omega_{q} b_{q \lambda}^{\dagger} b_{q \lambda}+\sum_{k \sigma} \epsilon_{k}^{c} c_{k \sigma}^{\dagger} c_{k \sigma}+\sum_{k \sigma} \epsilon_{k}^{v} v_{k \sigma}^{\dagger} v_{k \sigma} .
$$

The operators $c_{k \sigma}$ and $v_{k \sigma}$ denote annihilation operators of the free electrons in the conduction and valence bands, with quasimomentum $k$ and spin $\sigma$. The interaction Hamiltonian of the electrons and the photons in the dipole approximation is given by 29,30

$$
V=\sum_{\sigma, \sigma^{\prime}, \lambda} \sum_{p, q}\left[A_{p q}^{\sigma \sigma^{\prime} \lambda} c_{p+q \sigma}^{\dagger} v_{p \sigma^{\prime}} b_{q \lambda}+\text { H.c. }\right]
$$

where $A_{p q}^{\sigma \sigma^{\prime} \lambda}$ are the interaction matrix elements for the twoband model. ${ }^{1}$

Electrons excited above the gap are affected by the Coulomb interaction into two ways. First, the electronic density of states is modified, affecting the optical absorption, an effect that is known as the Sommerfeld enhancement. This enhancement decreases with the energy of the excitation, and far above the gap, it is small. For example, for bulk GaAs, the enhancement factor is of the order of unity for an excitation which is $50 \mathrm{meV}$ above the gap. ${ }^{29}$ Second, the Coulomb interaction contributes to spin relaxation rate $\gamma_{s}$ through the Bir-Pikus exchange mechanism, in addition to other mechanisms involving spin-orbit and electron-phonon interactions. ${ }^{1,4}$ As we discuss below, these dephasing rates are relatively small, and the qualitative effects which we find for the steady state $\left\langle\mathbf{S}^{2}\right\rangle$ should hold independently of the details of the dephasing mechanism.

\section{CAlCULATiON}

The total spin of the conduction electrons is given by $\mathbf{S}$ $=\Sigma_{k} \mathcal{S}_{k}$, where $\mathcal{S}_{k}=\Sigma_{\sigma \sigma^{\prime}} c_{k \sigma^{\dagger}}^{\dagger} \vec{\sigma}_{\sigma \sigma^{\prime}} c_{k \sigma^{\prime}}$. For the optical beam described above, photoexcited electrons have $\langle\mathbf{S}\rangle=0$, since the squeezed vacuum radiation is unpolarized. As for the variance $\left\langle\mathbf{S}^{2}\right\rangle$, only even orders in the field contribute and the second-order contribution is diagonal in $k$ space $\left\langle\mathbf{S}^{2}\right\rangle^{(2)}$ $=\Sigma_{k}\left\langle\mathcal{S}_{k}^{2}\right\rangle^{(2)}$ because it is a result of a single electron-hole pair excitation. Using the operator identity $\mathcal{S}_{k}^{2}=3\left[n_{k \uparrow}+n_{k \downarrow}\right]$ $-6 n_{k \uparrow} n_{k \downarrow}$, where $n_{k, \sigma}=c_{k \sigma}^{\dagger} c_{k \sigma}$, it follows that $\left\langle\mathbf{S}^{2}\right\rangle^{(2)}$ $=3 \Sigma_{k}\left[\left\langle n_{k \uparrow}\right\rangle^{(2)}+\left\langle n_{k \downarrow}\right\rangle^{(2)}\right]$, which is not sensitive to the photon correlations.

The next nonvanishing contribution is $\left\langle\mathbf{S}^{2}\right\rangle^{(4)}$, and we now turn to the calculation of its generation rate $C_{s}=d\left\langle\mathbf{S}^{2}\right\rangle^{(4)} / d t$, where 


$$
\left\langle\mathbf{S}^{2}(t)\right\rangle^{(4)}=\sum_{\{1, \ldots, 4\}} A_{p_{1}, q_{1}}^{\sigma_{1}, \sigma_{1}^{\prime}, \lambda_{1} *} A_{p_{2}, q_{2}}^{\sigma_{2}, \sigma_{2}^{\prime}, \lambda_{2} *} C_{p_{1}, q_{1}}^{p_{2}, q_{2} *} A_{p_{3}, q_{3}}^{\sigma_{3}, \sigma_{3}^{\prime}, \lambda_{3}} A_{p_{4}, q_{4}}^{\sigma_{4}, \sigma_{4}^{\prime}, \lambda_{4}} C_{p_{3}, q_{3}}^{p_{4}, q_{4}}\left\langle b_{q_{1} \lambda_{1}}^{\dagger} b_{q_{2} \lambda_{2}}^{\dagger} b_{q_{4} \lambda_{4}} b_{q_{3} \lambda_{3}}\right\rangle_{\mathrm{rad}}\left\langle 1,2\left|\mathbf{S}^{2}\right| 3,4\right\rangle_{e q}
$$

The average $\left\langle b_{q_{1} \lambda_{1}}^{\dagger} b_{q_{2} \lambda_{2}}^{\dagger} b_{q_{4} \lambda_{4}} b_{q_{3} \lambda_{3}}\right\rangle_{\text {rad }}$ is a property of the external field, and we have defined

$$
C_{p_{1}, q_{1}}^{p_{2}, q_{2}}=\frac{e^{i\left(\Delta \epsilon_{p_{1} q_{1}}+\Delta \epsilon_{p_{2} q_{2}}-\omega_{q_{1}}-\omega_{q_{2}}\right) t+2 \eta t}}{\Delta \epsilon_{p_{1} q_{1}}+\Delta \epsilon_{p_{2} q_{2}}-\omega_{q_{1}}-\omega_{q_{2}}-2 i \eta}\left[\frac{1}{\Delta \epsilon_{p_{1} q_{1}}-\omega_{q_{1}}-i \gamma_{p_{1}}}+\frac{1}{\Delta \epsilon_{p_{2} q_{2}}-\omega_{q_{2}}-i \gamma_{p_{2}}}\right],
$$

which is the second-order amplitude for the double photon absorption, and $e^{\eta t}$ is the adiabatic switching on factor. Here, $\gamma_{p}$ is the lifetime of the conduction electron states, ${ }^{32}$ and $\Delta \epsilon_{p q}=\epsilon_{p+q}^{c}-\epsilon_{p}^{v}$. In expression (3), the fermionic average is

$$
\left\langle 1,2\left|\mathbf{S}^{2}\right| 3,4\right\rangle_{e q}=\sum_{i} \sum_{k, s_{1}, s_{2}} \sum_{k^{\prime}, s_{1}^{\prime}, s_{2}^{\prime}} \sigma_{s_{1}, s_{2}}^{(i)} \sigma_{s_{1}^{\prime}, s_{2}^{\prime}}^{(i)}\left\langle c_{p_{1}+q_{1} \sigma_{1}} c_{p_{2}+q_{2} \sigma_{2}} c_{k s_{1}}^{\dagger} c_{k s_{2}} c_{k^{\prime} s_{1}^{\prime}}^{\dagger} c_{k^{\prime} s_{2}^{\prime}} c_{p_{3}+q_{3} \sigma_{3}}^{\dagger} c_{p_{4}+q_{4} \sigma_{4}}^{\dagger}\right\rangle_{e q}\left\langle v_{p_{1} \sigma_{1}^{\prime}}^{\dagger} v_{p_{2} \sigma_{2}^{\prime}}^{\dagger} v_{p_{3} \sigma_{3}^{\prime}} v_{p_{4} \sigma_{4}^{\prime}}\right\rangle_{e q},
$$

where $\langle\cdot\rangle_{e q}$ is assumed to be equilibrium at $T=0$.

A Wick decomposition of Eq. (3) contains contractions which contribute to the independent fluctuations $\Sigma_{k}\left\langle\mathcal{S}_{k}^{2}\right\rangle$ as well as contractions which contribute to the collective spinspin correlations $\left\langle\mathcal{S}_{k} \cdot \mathcal{S}_{k^{\prime}}\right\rangle$ with $k \neq k^{\prime}$. The latter can be further divided into two processes [Fig. 2(a)], in which (i) a singlet $(k \uparrow, k \downarrow)$ in the valence band is broken into two different momenta in the conduction band $\left(k+q, k+q^{\prime}\right)$ and (ii) two electrons with different momenta $\left(k_{1}, k_{2}\right)$ are excited into the conduction band with momenta $\left(k_{1}+q, k_{2}+q^{\prime}\right)$. Process (i) has considerably smaller rate with respect to (ii) because most of the phase space of final states cannot be reached with the typically small photon momentum. The ratio can be approximately estimated to be $(B / c k)^{2}$, where $k$ is the typical electron wave number. Therefore, in the following, we neglect the contribution of process (i).

For the generation rate $C_{s}$ of correlations due to process (ii), we use the corresponding contractions in Eq. (3), differentiating with respect to time and taking the limit $\eta \rightarrow 0$. This results in an energy conservation constraint for the entire process of exciting two electron-hole pairs. This process again has two parts [Fig. 2(b)]: one coming from normal contractions $\left\langle b^{\dagger} b\right\rangle^{2}$ and the other from anomalous contractions $|\langle b b\rangle|^{2}$ due to squeezing. For normal contractions, any

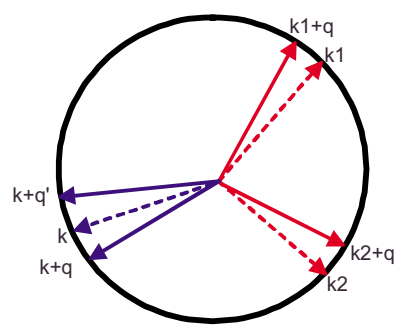

(a)

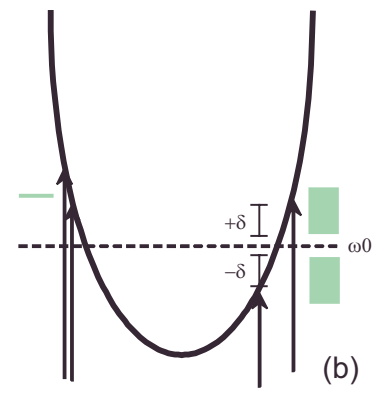

FIG. 2. (Color online) Correlation processes in the $k$ space. (a) The excitation of a singlet pair of momentum $k$ (blue dashed arrow) from the valence band into the conduction band (blue solid arrow) and excitation of two valence band electrons of different momenta (red). (b) Pair excitations associated with normal (left) photonic contractions (left-narrow green strip) and excitations associated with anomalous correlations (right-wide green strips, $\delta=\Delta \epsilon_{k}-\omega_{0}$ ). two spin components $\mathcal{S}_{k}, \mathcal{S}_{k^{\prime}}$ become correlated due to the absorption of two photons from the same mode $q$, obeying the energy conservation $\omega_{q}=\frac{1}{2}\left(\Delta \epsilon_{k}+\Delta \epsilon_{k^{\prime}}\right)$. In contrast, for anomalous contractions, only spin components which have symmetric energies $\Delta \epsilon_{k}+\Delta \epsilon_{k^{\prime}}=2 \omega_{0}$ become correlated. These are drawn out of a continuum of such pairs obeying $\omega_{q}+\omega_{q^{\prime}}=2 \omega_{0}$. The two processes are distributed very differently in phase space; however, both are significant since they turn out to have the same total phase space. These processes give the largest contribution to the generation rate of correlations.

\section{RESULTS AND DISCUSSION}

In the limit of $q \ll k$, the generation rate is given by (per unit volume)

$$
\begin{aligned}
C_{s}= & C_{s 0} \sum_{q} \int d \Delta \epsilon_{k} \rho\left(\Delta \epsilon_{k}\right) \rho\left(2 \omega_{0}-\Delta \epsilon_{k}\right)\left(N_{q}^{2}+\left|M_{q}^{(1)}\right|^{2}\right. \\
& \left.-\left|M_{q}^{(2)}\right|^{2}\right) \sqrt{\left.\left(\Delta \epsilon_{k}-\omega_{q}\right)^{2}+\left(\frac{\gamma}{2}\right)^{2}\right]^{2}},
\end{aligned}
$$

where $C_{s 0}=32 \pi^{2}|d|^{4} / 3 \hbar, d$ is the dipole matrix element, $\rho$ is the electronic density of states, $\gamma$ is the average of $\gamma_{p}$, and we assume $\left|M_{q \pm}^{(1,2)}\right|=\left|M_{q}^{(1,2)}\right|$, i.e., that the squeezed correlations for $(+)$ and $(-)$ polarizations differ only by phase. Since $\rho$ can be taken as constant in the excitation bandwidth, integrating Eq. (6) with respect to the electronic energy $\Delta \epsilon_{k}$ gives

$$
C_{s}=C_{s 0} \rho\left(\omega_{0}\right)^{2} \frac{4 \pi}{\gamma} \sum_{q}\left(N_{q}^{2}+\left|M_{q}^{(1)}\right|^{2}-\left|M_{q}^{(2)}\right|^{2}\right) .
$$

This result shows that increased (decreased) fluctuations of the Stokes parameter $p_{3}$ (cf. Fig. 1) lead to increased (decreased) spin correlations. Positive spin correlations induced by absorbing photons from the same mode are enhanced by absorbing squeezed photons with the samepolarization modes and reduced by the squeezing of the opposite polarizations (cf. Fig. 3). In contrast to the atomic optical case, the fourth-order processes generate inevitable 


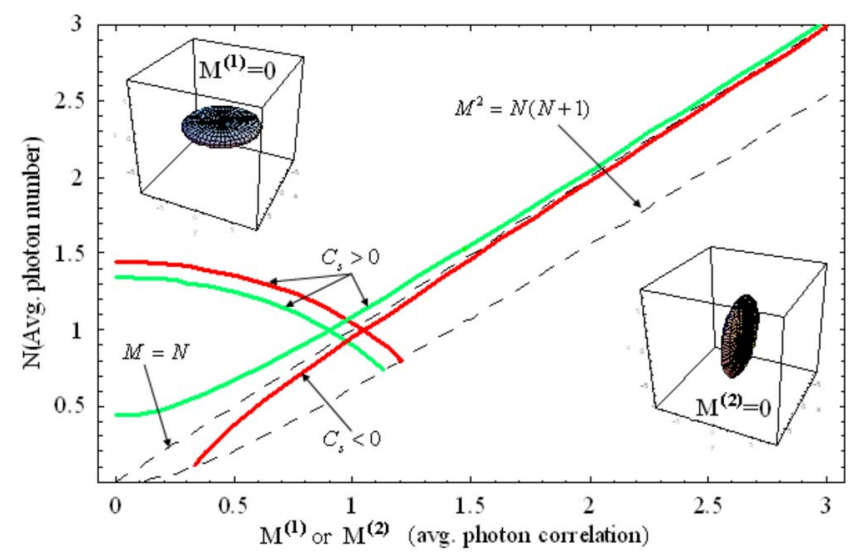

FIG. 3. (Color online) Curves of equal collective spin correlation in the parameters of average occupation $(N)$ and squeezing $(M)$. Cases drawn for enhanced (circle arcs) and reduced (hyperbola arcs) spin correlations, corresponding to $M^{(2)}=0$ and $M^{(1)}=0$, respectively. The colors signify different values of $C_{s}$. The dashed lines separate classical from quantum light (refer to the text). Insets: patterns of fluctuations of the Stokes vector leading to the enhanced and reduced spin correlations.

correlations $\left(N_{q}\right)$, even when the light is not squeezed. Therefore spin correlations can become negative only when the squeezing is nonclassical, i.e., $M^{(2)}>N$. The maximal negative spin correlations will be reached for a pure squeezed state with $M^{(2)}=\sqrt{N(N+1)},{ }^{33}$ in which case $C_{s} \sim-N$. Remarkably, this result contrasts most of the quantum optical effects which are observable only for very weak light, $N$ $\ll 1$, e.g., the effect of squeezing in two-photon absorption. ${ }^{34}$

It is instructive to consider curves of equal spin correlations (cf. Fig. 3). The dashed lines divide the figure to regions of classical light $M^{(1,2)} \leq N$, quantum light $N<M^{(1,2)}$ $\leq \sqrt{N(N+1)}$, and an unphysical region ${ }^{33} M^{(1,2)}>\sqrt{N(N+1)}$. For opposite-polarization squeezing (lying ellipsoid), the behavior of the spin correlations is essentially different from the case of same-polarization squeezing (standing ellipsoid). Only in the first case, the curves are confined to either the classical side $\left(C_{s}>0\right)$ or the quantum side $\left(C_{s}<0\right)$ of the diagram, with the $C_{s}=0$ being a separatrix between the two regimes. The possibility to completely eliminate the inevitable spin correlations induced by unsqueezed light may be useful for observing other sources of spin correlations such as contributions from exciton-exciton interactions.

It is useful to define a reduced density matrix $\rho_{\alpha \beta}^{\left(k, k^{\prime}\right)}$ for a pair of spins $\mathcal{S}_{k}, \mathcal{S}_{k^{\prime}}$, where $\alpha, \beta$ run over the singlet $(|0,0\rangle)$ and three triplet basis states $(|1,0\rangle,|1, \pm 1\rangle)$. In this basis, it can be easily shown that for negative spin correlations $C_{s}$ $<0$, the diagonal elements $\rho_{\alpha} \equiv \rho_{\alpha, \alpha}$ obey

$$
\sum_{k, k^{\prime}} \rho_{0,0}^{\left(k, k^{\prime}\right)}>\frac{1}{3} \sum_{k, k^{\prime}}\left(\rho_{1,-1}^{\left(k, k^{\prime}\right)}+\rho_{1,0}^{\left(k, k^{\prime}\right)}+\rho_{1,1}^{\left(k, k^{\prime}\right)}\right),
$$

which means that for the electronic state generated by nonclassical light, there is a preponderance of the singlet component in the pairwise spin correlations.

Experimentally, long spin dephasing times of the average spin have been measured by different techniques for different materials and experimental conditions., ${ }^{49}$ These include $n$-GaAs quantum wells with different widths, ${ }^{5}$ doping levels, ${ }^{7,17}$ materials, ${ }^{18,20}$ as well as bulk $n$-GaAs. ${ }^{21}$ In these experiments, spin lifetimes ranging from hundreds of picoseconds to tens of nanoseconds have been reported. In some cases, the long lifetimes were measured even in room temperature. ${ }^{19}$

Recently, also the fluctuations of the spin $\left\langle\mathbf{S}^{2}\right\rangle$ have been measured from the variance of the magnetic moment of the sample in a Faraday rotation setup. ${ }^{31}$ In order to enhance the correlation effects which we discuss here, it is advantageous to use samples with long spin lifetime, such as in $n$-type bulk GaAs. ${ }^{17}$ Spin flip times of the photoexcited holes are much faster compared to the electrons, ${ }^{4}$ and therefore, their contribution to the collective spin correlations is expected to be small. In such an experiment, enhancement or reduction of spin-spin correlations should be measurable from the difference of $\left\langle\mathbf{S}^{2}\right\rangle$ with squeezed and unsqueezed light, as can be seen from Eq. (7). A clear-cut evidence for the successful storing of optical correlations in the electron spins would be a signal of negative photon polarization correlations in the hot electron luminescence, appearing when the squeezing is turned on.

\section{ACKNOWLEDGMENTS}

We would like to thank I. Bar-Joseph and I. Neder for valuable discussions. *eran.ginossar@weizmann.ac.il

${ }^{1}$ F. Meier and B. P. Zakharchenya, Optical Orientation (NorthHolland, Amsterdam, 1984).

${ }^{2}$ V. D. Dymnikov, M. I. D’yakonov, and N. I. Perel', Sov. Phys. JETP 44, 1252 (1976).

${ }^{3}$ V. F. Gantmakher and Y. B. Levinson, Carrier Scattering in Metals and Semiconductors (Elseviers Science, New York, 1987), Chap. 4.

${ }^{4}$ I. Žutić, J. Fabian, and S. Das Sarma, Rev. Mod. Phys. 76, 323 (2004).
${ }^{5}$ S. Pfalz, R. Winkler, T. Nowitzki, D. Reuter, A. D. Wieck, D. Hägele, and M. Oestreich, Phys. Rev. B 71, 165305 (2005).

${ }^{6}$ D. K. Young, J. A. Gupta, E. Johnston-Halperin, R. Epstein, Y. Kato, and D. D. Awschalom, Semicond. Sci. Technol. 17, 275 (2002).

${ }^{7}$ M. Oestreich, M. Bender, J. Hübner, D. Hägele, W. W. Rühle, Th. Hartmann, P. J. Klar, W. Heimbrodt, M. Lampalzer, K. Volz, and W. Stolz, Semicond. Sci. Technol. 17, 285 (2002).

${ }^{8}$ G. M. Palma and P. L. Knight, Phys. Rev. A 39, 1962 (1989).

${ }^{9}$ G. S. Agarwal and R. R. Puri, Phys. Rev. A 41, 3782 (1990). 
${ }^{10}$ E. Ginossar and S. Levit, Phys. Rev. B 72, 075333 (2005).

${ }^{11}$ A. Kuzmich, K. Mølmer, and E. S. Polzik, Phys. Rev. Lett. 79, 4782 (1997).

${ }^{12}$ A. E. Kozhekin, K. Mølmer, and E. Polzik, Phys. Rev. A 62, 033809 (2000).

${ }^{13}$ A. Serafini, M. Paternostro, M. S. Kim, and S. Bose, Phys. Rev. A 73, 022312 (2006).

${ }^{14}$ M. D. Lukin, S. F. Yelin, and M. Fleischhauer, Phys. Rev. Lett. 84, 4232 (2000).

${ }^{15}$ J. Hald, J. L. Sørensen, C. Schori, and E. S. Polzik, Phys. Rev. Lett. 83, 1319 (1999).

${ }^{16}$ K. Hammerer, E. S. Polzik, and J. I. Cirac, Phys. Rev. A 72, 052313 (2005).

${ }^{17}$ J. M. Kikkawa and D. D. Awschalom, Phys. Rev. Lett. 80, 4313 (1998).

${ }^{18}$ Y. Ohno, R. Terauchi, T. Adachi, F. Matsukura, and H. Ohno, Phys. Rev. Lett. 83, 4196 (1999).

${ }^{19}$ S. A. Wolf, D. D. Awschalom, R. A. Buhrman, J. M. Daughton, S. von Molnár, M. L. Roukes, A. Y. Chtchelkanova, and D. M. Treger, Science 294, 1488 (2001).

${ }^{20}$ B. Beschoten, E. Johnston-Halperin, D. K. Young, M. Poggio, J. E. Grimaldi, S. Keller, S. P. DenBaars, U. K. Mishra, E. L. Hu, and D. D. Awschalom, Phys. Rev. B 63, 121202(R) (2001).

${ }^{21}$ R. I. Dzhioev, K. V. Kavokin, V. L. Korenev, M. V. Lazarev, N. K. Poletaev, B. P. Zakharchenya, E. A. Stinaff, D. Gammon, A.
S. Bracker, and M. E. Ware, Phys. Rev. Lett. 93, 216402 (2004).

${ }^{22}$ L. Bányai, D. B. Tran Thoai, E. Reitsamer, H. Haug, D. Steinbach, M. U. Wehner, M. Wegener, T. Marschner, and W. Stolz, Phys. Rev. Lett. 75, 2188 (1995).

${ }^{23}$ G. Bartels, V. M. Axt, K. Victor, A. Stahl, P. Leisching, and K. Köhler, Phys. Rev. B 51, 11217 (1995).

${ }^{24}$ E. Collett, Polarized Light: Fundamentals and Applications (Dekker, New York, 1993).

${ }^{25}$ N. Korolkova, G. Leuchs, R. Loudon, T. C. Ralph, and C. Silberhorn, Phys. Rev. A 65, 052306 (2002).

${ }^{26}$ M. J. Collet and R. Loudon, J. Opt. Soc. Am. B 4, 1525 (1987).

${ }^{27}$ Yanhua Shih, Rep. Prog. Phys. 66, 1009 (2003).

${ }^{28}$ W. P. Grice and I. A. Walmsley, Phys. Rev. A 56, 1627 (1997).

${ }^{29} \mathrm{H}$. Haug and S. W. Koch, Quantum Theory of the Optical and Electronic Properties of Semiconductors (World Scientific, Singapore, 1990).

${ }^{30}$ G. Khitrova, H. M. Gibbs, F. Jahnke, M. Kira, and S. W. Koch, Rev. Mod. Phys. 71, 1591 (1999).

${ }^{31}$ M. Oestreich, M. Römer, R. J. Haug, and D. Hägele, Phys. Rev. Lett. 95, 216603 (2005).

${ }^{32}$ T. Takagahara, E. Hanamura, and R. Kubo, J. Phys. Soc. Jpn. 43, 802 (1977).

${ }^{33}$ C. W. Gardiner, Phys. Rev. Lett. 56, 1917 (1986).

${ }^{34}$ N. P. Georgiades, E. S. Polzik, K. Edamatsu, H. J. Kimble, and A. S. Parkins, Phys. Rev. Lett. 75, 3426 (1995). 